\title{
Aeroassisted Orbital Transfer Trajectory Optimization Considering Thermal Protection System Mass
}

\author{
Christian Gogu*, , Taiki Matsumura*,‡, Raphael T. Haftka ${ }^{\S}$ and Anil V. Rao \\ University of Florida, Gainesville, Florida 32611
}

\begin{abstract}
Aeroassisted orbital transfer is recognized as a potential technology to enhance the operational responsiveness of space with significant fuel savings. In order to use aerodynamic forces resulting from the flight through the atmosphere, however, considerable thermal protection is required, thereby potentially decreasing the savings in mass achieved by lowering the fuel consumption. In this paper the relationship between achievable fuel savings and thermal protection system (TPS) size is investigated by coupling these two disciplines through a single coupling parameter, the maximum heating rate constraint. The optimal solution that minimizes the total mass of the vehicle (fuel and TPS) is then determined. A trajectory optimization procedure and a TPS mass estimation model are then applied to a problem where it is desired to transfer a vehicle using an aeroassisted maneuver between two low earth orbits with a specified inclination change. All trajectory parameters, including de-orbit, boost, and recircularization impulses, are optimized and the thermal protection system is sized with ablative and reusable materials. It is found that the aeroassisted maneuver maintains a overall mass advantage over an all propulsive maneuver. It is also found that the minimum overall mass (fuel and TPS) is achieved when no heating rate constraint is imposed, which is also the scenario that minimizes the fuel consumption alone.
\end{abstract}

\section{Nomenclature}

$\begin{array}{ll}C_{D} & \text { coefficient of drag } \\ C_{D 0} & \text { zero-lift coefficient of drag } \\ C_{L} & \text { coefficient of lift } \\ C_{L, \alpha} & \text { derivative of } C_{L} \text { with respect to } \alpha \\ C_{L, \text { max }} & \text { maximum coefficient of lift } \\ D & \text { drag acceleration } \\ g & \text { gravitational acceleration } \\ g_{0} & \text { gravitational acceleration at sea level } \\ h_{a} & \text { heat of ablation } \\ h_{a t m} & \text { altitude of sensable atmosphere } \\ h_{v} & \text { heat of vipolarization } \\ h_{0} & \text { altitude of low earth orbit } \\ i & \text { inclination } \\ i_{d} & \text { desired inclination change } \\ I_{s p} & \text { specific impulse } \\ J & \text { performance index } \\ K & \text { drag polar parameter } \\ L & \text { lift acceleration }\end{array}$

\footnotetext{
${ }^{*}$ The two authors marked with * share first authorship for this paper and are presented in alphabetical order

${ }^{\dagger}$ Graduate Research Assistant, Department of Mechanical and Aerospace Engineering, P.O.box 116250. AIAA Student Member.

${ }^{\ddagger}$ Visiting researcher at the University of Florida. Associate Engineer, Japan Aerospace Exploration Agency (JAXA).

$\S$ Distinguished Professor, Department of Mechanical and Aerospace Engineering, P.O.box 116250. AIAA Fellow.

『Assistant Professor. Department of Mechanical and Aerospace Engineering, P.O.box 116250. Corresponding Author. E-mail: anilvrao@ufl.edu
} 


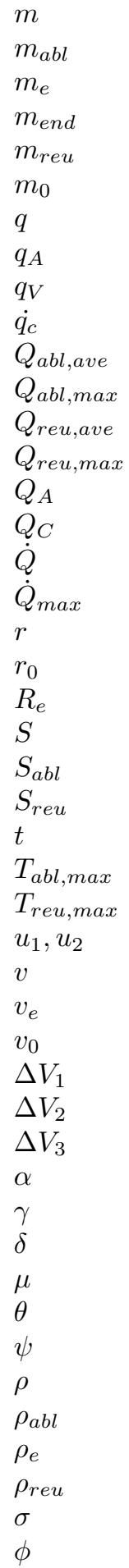

Subscripts

$f$ vehicle mass

mass of ablative TPS material

vehicle empty mass

vehicle mass at the end of the mission (after recircularization)

mass of reusable TPS material

vehicle gross mass

dynamic pressure

heat of ablation

heat of vaporization

heating rate to keep TPS surface its ablation temperature

average total heat load of ablative section

maximum total heat load of ablative section (heat load at stagnation point)

average total heat load in reusable TPS section

maximum total heat load in reusable TPS section

ablation heat load

conduction heat load

heating rate

maximum stagnation point heating rate

radius

radius of low earth orbit (LEO)

radius of Earth

vehicle reference area

area of ablative section

area of reusable section

time

maximum thickness of ablative TPS material

maximum thickness of reusable TPS material

control variables

velocity

circular orbit speed at surface of the Earth

circular orbit speed at Low Earth Orbit

magnitude of impulse for deorbit

magnitude of impulse to raise the apogee of the transfer orbit

magnitude of impulse for recircularization

angle of attack

flight path angle

recession thickness of ablative TPS material

gravitational parameter

longitude angle

heading angle

air density

density of ablative material

air density at sea level

density of reusable material

bank angle

latitude angle

final value of atmospheric flight

initial value of atmospheric flight 


\section{Introduction}

Vehicles such as the Orbital Space Plane (OSP) have been recognized in recent years as concepts that greatly increase the operational responsiveness of space. ${ }^{1}$ In particular, an OSP-type vehicle can potentially provide both efficient regular transportation and safe return of crew members in case of emergency on orbit. (i.e., catastrophic failure of life support systems of the International Space Station (ISS)). To improve operational responsiveness, it is useful for the OSP to have high crossrange capability during atmospheric flight (i.e., the OSP must be capable of aeroassisted orbital transfer). In addition to improving the reachable footprint, the aeroassisted technology potentially enhances an on-orbit maneuver, such as orbital transfer and orbital plane change, and lowers its fuel consumption, thereby reducing overall mission cost as compared to an all-propulsive orbital transfer.

The idea of aeroassisted orbital transfer has been studied extensively in the literature and much of the early work is summarized in Refs. 2 and 3. More recently optimal trajectories that are subject to high heating rate constraints have been studied in Refs. 4-7. In these past studies several types of aeroassisted maneuvers, such as aerocruise with propulsive maneuvers and aeroglide without propulsive maneuvers, have been discussed and it has been found that the heating rate constraint is one of the key parameters to determine the performance of the aeroassisted orbital transfer (i.e., capacity of inclination change). In addition, multiple-pass aeroassisted orbital transfer from geostationary orbit to low earth orbit with desired inclination change subject to heating rate constraints has been studied in Ref. 8 and 9. It was shown that the heating rate constraint is also the key parameter for fuel saving performance. Finally, in the somewhat more practical study of Ref. 10, the feasibility of multiple-pass aeroassisted orbital transfer with inclination change for the X-37 vehicle, was discussed.

In this study the simultaneous trajectory optimization and TPS design is investigated to determine the minimum mass of a high lift-to-drag $(L / D)$ ratio aeroassisted orbital transfer vehicle (AOTV) for a problem with orbital plane change. The desired inclination change is accomplished using an aeroglide (i.e., nonthrusting) maneuver during atmospheric flight. The trajectory optimization and the TPS design are coupled through a single coupling parameter, the maximum heating rate constraint. While it may be evident that the trajectory impacts TPS design, it may be less intuitive that a reverse coupling exists as well, i.e., that the TPS design impacts the trajectory because changes in the TPS size and shape change the mass and shape of the vehicle. By fixing the total mass, fuel, and exterior shape of the vehicle, this reverse coupling is eliminated from the problem. i.e., any variations in the TPS or fuel savings will change payload size and mass. It is also assumed that no other coupling between the trajectory and TPS design other than the heating rate constraint exists. By using this single coupling parameter we can solve the multi-disciplinary design optimization (MDO) problem sequentially. First the optimal trajectory is determined that minimizes the fuel consumption to achieve the desired inclination change for various heating rate constraints. For each trajectory obtained, the size of the TPS is subsequently determined. Finally we find the optimum design by finding the heating rate corresponding to minimum combined weight of the fuel and TPS.

The trajectory optimization problem is posed as a nonlinear optimal control problem and is solved using the software GPOCS ${ }^{11}$ which is an implementation of the Gauss Pseudospectral method (GPM) ${ }^{12}$ For the second step relative to the thermal shield design, the TPS sizing is carried out using both ablative and reusable materials is applied. To calculate the required thickness of the TPS we use a simplified analysis tool called the virtual ablation method ${ }^{13}$ for the ablative material and a finite element heat transfer analysis for the reusable material.

\section{Trajectory Optimization for Aeroassisted Orbital Transfer}

Consider the following orbital transfer problem for a vehicle modeled as a point mass over a spherical nonrotating Earth. The transfer is started with a deorbit impulse from an initial circular low-Earth orbit (LEO) with altitude $h_{0}$ resulting in an elliptic transfer orbit. The initial LEO and the elliptic orbit are assumed to lie in the equatorial plane. The elliptic transfer orbit terminates at an altitude $h_{\text {atm }}$ where $h_{\text {atm }}$ is the altitude at the edge of the sensable atmosphere (i.e., the altitude at which the atmosphere is first sensable by on-board instrumentation). The vehicle then flies with aerodynamic control through the atmosphere under the influence of drag, lift, and gravity such that the atmospheric flight segment terminates at an altitude of $h_{\mathrm{atm}}$. It is assumed that all inclination change is achieved purely by the aerodynamic force during atmospheric flight. Upon atmospheric exit, a second impulse is applied to raise the apogee of the 
resulting transfer orbit. After the application of the second impulse, the vehicle flies along another elliptic transfer orbit until apogee is attained. Upon reaching apogee, a third impulse is applied to recircularize the orbit. The event sequence for the trajectory optimization is given as

Deorbit Impulse $\longrightarrow$ Keplerian Flight $\longrightarrow$ Atmospheric Flight
$\longrightarrow$ Boost Impulse $\longrightarrow$ Keplerian Flight $\longrightarrow$ Circularization Impulse

Finally, a schematic of the orbital transfer corresponding to the trajectory event sequence is shown in Fig. 1.

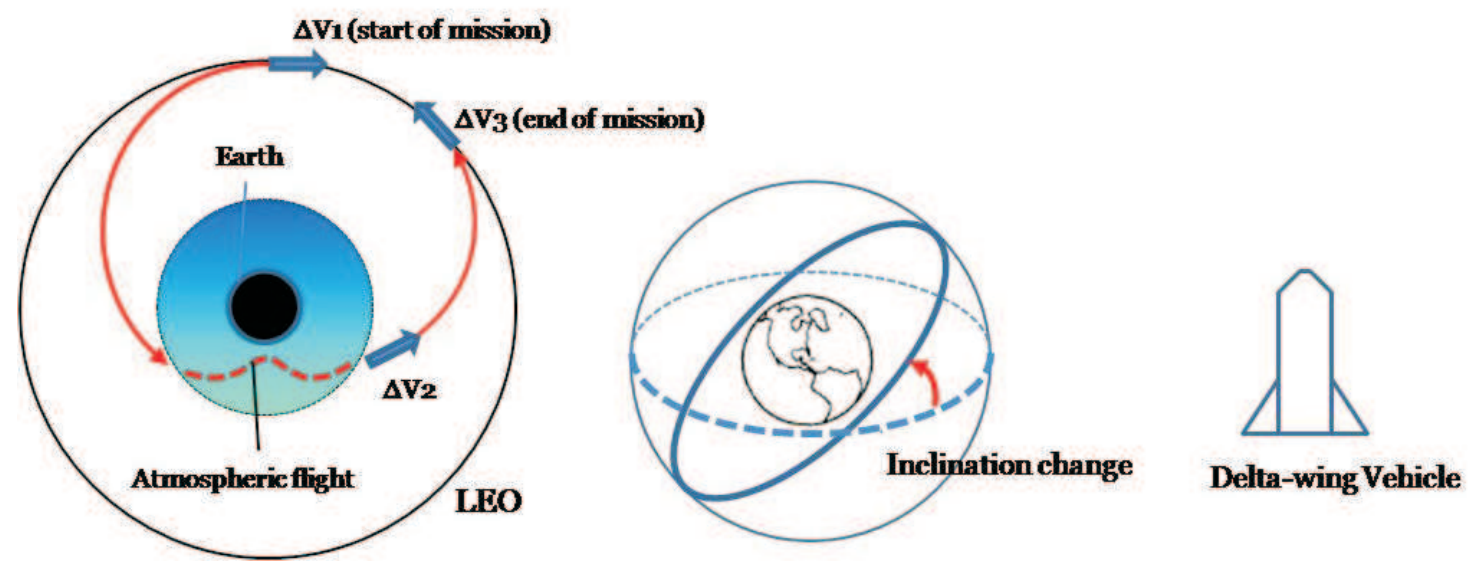

Figure 1. Schematic of the mission scenario of the AOTV.

\section{II.A. Physical and Vehicle Models}

For this study the vehicle is modeled as a point mass in motion over a spherical nonrotating Earth. During exo-atmospheric flight, the vehicle is assumed to be under the influence of spherical gravity with the exception of three specific instants of time where impulsive thrust is applied. The first impulse, $\Delta V_{1}$, is applied from the initial circular orbit of radius $r_{0}=h_{0}+R_{e}$ (where $R_{e}$ is the radius of the Earth), thereby resulting in an elliptic transfer orbit that intersects the sensable atmosphere. The second impulse, $\Delta V_{2}$, is applied upon exit from the atmosphere where the radius, speed, and flight path angle are given, respectively, as $r_{f}, v_{f}$ and $\gamma_{f}$. The second impulse raises the apogee of the ascending elliptical orbit to the initial LEO radius, $r_{0}$. Because after atmospheric exit, (i.e., right after $\Delta V_{2}$ is applied and before $\Delta V_{3}$ is applied), it is known that the energy and angular momentum are conserved. As a result, $\Delta V_{2}$ is given as

$$
\Delta V_{2}=\left[\frac{2 \mu\left(1 / r_{f}-1 / r_{0}\right)}{1-\left(r_{f} / r_{0}\right)^{2} \cos ^{2} \gamma_{f}}\right]^{1 / 2}-v_{f}
$$

Similarly, the third impulse, $\Delta V_{3}$, is applied at the apogee of the post-atmospheric space flight segment to recircularize the elliptical orbit to a radius of $r_{0}$ (where the circular orbit speed is $v_{0}$ ). The third impulse is then obtained as

$$
\Delta V_{3}=v_{0}-\frac{r_{f}}{r_{0}}\left[\frac{2 \mu\left(1 / r_{f}-1 / r_{0}\right)}{1-\left(r_{f} / r_{0}\right)^{2} \cos ^{2} \gamma_{f}}\right]^{1 / 2} \cos \gamma_{f}
$$

During atmospheric flight, the vehicle is assumed to be under the influence of drag, lift, and gravity. The three degree-of-freedom differential equations of motion are given in spherical coordinates ${ }^{14}$ as

$$
\begin{aligned}
\frac{d r}{d t} & =v \sin \gamma \\
\frac{d \theta}{d t} & =\frac{v \cos \gamma \cos \psi}{r \cos \phi} \\
\frac{d \phi}{d t} & =\frac{v \cos \gamma \sin \psi}{r}
\end{aligned}
$$




$$
\begin{aligned}
\frac{d v}{d t} & =-D-g \sin \gamma \\
\frac{d \gamma}{d t} & =\frac{1}{v}\left[L \cos \sigma-\left(g-\frac{v^{2}}{r}\right) \cos \gamma\right] \\
\frac{d \psi}{d t} & =\frac{1}{v}\left[\frac{L \sin \sigma}{\cos \gamma}-\frac{v^{2}}{r} \cos \gamma \cos \psi \tan \phi\right]
\end{aligned}
$$

Next, the magnitude of the gravitational acceleration is given as

$$
g=\mu / r^{2}
$$

The drag and lift specific forces are denoted as $D$ and $L$, respectively, and are given, respectively, as

$$
\begin{aligned}
D & =q S C_{D} / m \\
L & =q S C_{L} / m
\end{aligned}
$$

where $q=\rho v^{2} / 2$ is the dynamic pressure. The atmospheric density is calculated from a 1962 U.S. Standard Atmosphere model. The aerodynamic model used in this study is a drag polar of the form

$$
\begin{aligned}
C_{L} & =C_{L, \alpha} \alpha \\
C_{D} & =C_{D 0}+K C_{L}^{2}
\end{aligned}
$$

It is assumed in this study that the vehicle is lift-limited such that $C_{L} \in\left[0, C_{L, \max }\right]$. The aerodynamic and physical model of the high $L / D$ delta-wing vehicle and the numerical values of all constants used in this study are adapted from Ref. 7 and shown in Table.1.

Table 1. Aerodynamic data, vehicle data and physical data of AOTV problem.

\begin{tabular}{lll}
\hline Quantity & & Numerical value \\
\hline$m_{0}$ & vehicle gross mass & $4898.7 \mathrm{~kg}$ \\
$m_{e}$ & vehicle empty mass & $2283.9 \mathrm{~kg}$ \\
& vehicle fuel mass & $2614.8 \mathrm{~kg}$ \\
$R_{e}$ & radius of Earth & $6378.4 \mathrm{~km}$ \\
$\mu$ & gravitational parameter & $398970 \mathrm{~km}^{2} / \mathrm{s}^{2}$ \\
$S$ & vehicle reference area & $11.69 \mathrm{~m}^{2}$ \\
$C_{D 0}$ & zero-lift coefficient of drag & 0.032 \\
$K$ & drag polar parameter & 1.4 \\
$C_{L, \alpha}$ & derivative of $C_{L}$ with respect to $\alpha$ & 0.5699 \\
$C_{L, \max }$ & maximum coefficient of lift & 0.4 \\
$h_{0}$ & initial LEO altitude & $185.2 \mathrm{~km}$ \\
$h_{\text {atm }}$ & altitude of sensable atmosphere & $129.6 \mathrm{~km}$ \\
$I_{s p}$ & specific impulse & $310 \mathrm{~s}$ \\
$\overline{\bar{Q}}$ & heating rate constant & $19987.44 \mathrm{~W} / \mathrm{m}^{2}$ \\
\hline
\end{tabular}

\section{II.B. Path Constraints and Boundary Conditions}

During atmospheric flight, the following path constraints are imposed on the vehicle and are taken from Ref. 8. First, an inequality path constraint on the stagnation point heating rate, $\dot{Q}$, is imposed. The model for the stagnation point heating rate is taken from Ref. 16 and is given as

$$
\dot{Q}=\dot{\bar{Q}}\left(\frac{\rho}{\rho_{e}}\right)^{1 / 2}\left(\frac{v}{v_{e}}\right)^{3.15}
$$


where $\dot{\bar{Q}}$ is a constant whose numerical value is given in Table 1 . Note that this model accounts only for convective heating. We neglect the radiative heating contribution in this study. The heating rate constraint is then given as

$$
\dot{Q} \leq \dot{Q}_{\max }
$$

Next, because of the aliasing problems associated with using the bank angle, the following controls are used in place of the angle of attack and bank angle:

$$
\begin{aligned}
& u_{1}=-C_{L} \sin \sigma \\
& u_{2}=-C_{L} \cos \sigma
\end{aligned}
$$

Then, in terms of $u_{1}$ and $u_{2}$, we have

$$
C_{L}=\sqrt{u_{1}^{2}+u_{2}^{2}}
$$

The following inequality path constraint is then imposed on $u_{1}$ and $u_{2}$ during atmospheric flight:

$$
\sqrt{u_{1}^{2}+u_{2}^{2}} \leq C_{L, \max }
$$

Next, to ensure that the vehicle can reach apogee after exiting the atmosphere, the flight path angle must be positive at atmospheric exit, i.e.,

$$
\gamma_{f} \geq 0
$$

Also, the mission goal is to achieve the desired inclination change, $i_{d}$. Consequently the following boundary condition is applied at the atmospheric exit:

$$
i_{f}=i_{d}
$$

where the inclination is computed using the latitude and heading angle as

$$
\cos i=\cos \phi \cos \psi
$$

\section{II.C. Objective Function}

In order to get a optimal trajectory which minimizes the fuel consumption, the objective function is set to maximize the final vehicle mass, $m_{\text {end }}$, subject to the path constraints and for a given final inclination change. The performance index is described as

$$
J=-m_{\text {end }}
$$

$m_{\text {end }}$ is the final vehicle mass after the recircularization to the initial LEO and obtained by calculating the fuel consumption of each impulse as

$$
m_{\text {end }}=m_{0} \exp \left(-\Delta V_{1} / g_{0} I_{s p}\right) \exp \left(-\Delta V_{2} / g_{0} I_{s p}\right) \exp \left(-\Delta V_{3} / g_{0} I_{s p}\right)
$$

\section{II.D. Numerical Optimization}

The aeroassisted orbital plane change problem is solved using the optimal control software GPOCS ${ }^{11}$ which is an implementation of the Gauss pseudospectral method (GPM) developed in Ref. 12. In this study a single-phase optimal control problem was posed using 50 nodes (i.e., 50 Legendre-Gauss points). GPOCS uses the TOMLAB ${ }^{17}$ version of the sparse NLP solver SNOPT. ${ }^{18}$

\section{Estimating Mass of Thermal Protection System}

In our mission scenario, the vehicle will enter Earth's atmosphere at hypersonic speed and meet severe heating environment. Some sections of the vehicle will experience very high heating rates requiring an ablative heat shield since reusable TPS materials have operational temperature limits. Ablative TPS materials accommodate high heating rates and heat loads through phase change and mass loss (i.e., pyrolysis, charring and recession). However, ablative materials have a disadvantage in terms of mass because the densities of ablative materials are higher than those of reusable materials. We therefore apply an ablative TPS material in high heating environments and a reusable material in low heating environments. For ablative material 
we considered two flight-certified materials, SLA-561 and AVCOAT-5026-39H/CG. ${ }^{19}$ SLA-561 which was developed and used as an ablative heat shield on the Viking mission. SLA-561 has lower density and is composed of a silicone resin with cork, phenolic microballoon, silica microballoon, and refractory fiber fillers. AVCOAT-5026-39H/CG is an older material, used for the Apollo mission, but it is still considered as a potential candidate for the TPS of the CEV (Crew Exploration Vehicle) of the Orion project. The AVCOAT-5026-39H/CG consists of an epoxy-novalac resin reinforced with quartz fibers and phenolic microballoons. For the reusable material we chose the LI-900 rigidized ceramic insulation tiles which are used over the major portion of the windward surface (bottom side) of the Space Shuttle orbiter. The material properties of these materials are taken from Refs. 13,20,21 and some of the relevant properties are shown in Table 2. Note that in our models we used temperature dependent material properties for the thermal conductivity and specific heat. In the following section, we present the procedure to estimate mass of the TPS, which consists of TPS area definition, required thickness sizing, and total TPS mass calculation.

Table 2. Material properties

[Ablative material]

\begin{tabular}{lll}
\hline Material & Quantity & Numerical value \\
\hline SLA-561 & Heat of ablation & $5.41 \times 10^{7} \mathrm{~J} / \mathrm{kg}$ \\
& Density (virgin material) & $2.64 \times 10^{2} \mathrm{~kg} / \mathrm{m}^{3}$ \\
& Thermal conductivity @295K & $5.92 \times 10^{-2} \mathrm{~W} / \mathrm{mK}$ \\
& Specific heat @295K & $1.17 \times 10^{3} \mathrm{~J} / \mathrm{kgK}$ \\
& Ablation temperature & $922 \mathrm{~K}$ \\
AVCOAT-5026-39H/CG & Heat of ablation & $1.38 \times 10^{7} \mathrm{~J} / \mathrm{kg}$ \\
& Density (virgin material) & $5.29 \times 10^{2} \mathrm{~kg} / \mathrm{m}^{3}$ \\
& Thermal conductivity @295K & $2.97 \times 10^{-1} \mathrm{~W} / \mathrm{mK}$ \\
& Specific heat @295K & $1.70 \times 10^{3} \mathrm{~J} / \mathrm{kgK}$ \\
& Ablation temperature & $922 \mathrm{~K}$ \\
\hline
\end{tabular}

[Reusable material]

\begin{tabular}{lll}
\hline Material & Quantity & Numerical value \\
\hline LI-900 & Density & $1.442 \times 10^{2} \mathrm{~kg} / \mathrm{m}^{3}$ \\
& Thermal conductivity @295K & $4.76 \times 10^{-2} \mathrm{~W} / \mathrm{mK}$ \\
& Specific heat @295K & $6.28 \times 10^{2} \mathrm{~J} / \mathrm{kgK}$ \\
& Maximum operational temperature & $1.59 \times 10^{3} \mathrm{~K}$ \\
\hline
\end{tabular}

\section{III.A. Area Definition of TPS materials}

An area definition of TPS with ablative materials, SLA-561 or AVCOAT-5026-39H/CG, and reusable material, LI-900, is generated in the following manner. First for a given AOTV trajectory the peak heating rate is assumed to be calculated at the nose stagnation point by Eq. (14). The variations of the heating rate along the windward streamline of the vehicle are modeled by the normalized heating rate distribution shown in Fig. 2, which was obtained on a generic space plane vehicle geometry ${ }^{22}$ in an optimization study of a high $L / D$ vehicle. Note that to simplify the problem the normalized heating rate distribution is assumed to be applied uniformly over the entire vehicle (not varying along the width of the vehicle) and not to vary with the angle-of-attack during the atmospheric flight. TPS materials are mapped to each surface location using an appropriate heating rate range for each TPS material. The concept of the area definition is drawn schematically in Fig. 3. According to the radiative thermal equilibrium temperature, the maximum operational heating rate of the LI-900 is calculated as $31.9 \mathrm{~W} / \mathrm{cm}^{2}$. Thus the heating rate ranges of the vehicle used in this study are given in Table 3.

The shape of the vehicle is assumed to be approximately similar to the X-37 vehicle. By using the vehicle length, width, ${ }^{23}$ and the geometry picture, we approximate the bottom surface area of X-37. And then by using a ratio of reference areas the bottom surface area of the vehicle is obtained as $42.5 \mathrm{~m}^{2} .{ }^{23}$ 
Table 3. Heating rate ranges for material selection

Heating rate ranges material selection

$\dot{Q}>31.9 \mathrm{~W} / \mathrm{cm}^{2} \quad$ ablative material

$\dot{Q} \leq 31.9 \mathrm{~W} / \mathrm{cm}^{2} \quad$ reusable material (LI-900)

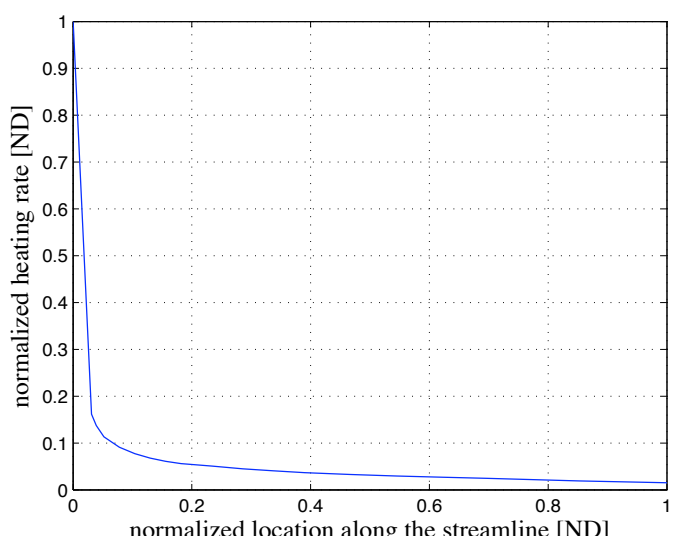

Figure 2. The normalized heating rate distribution curb.
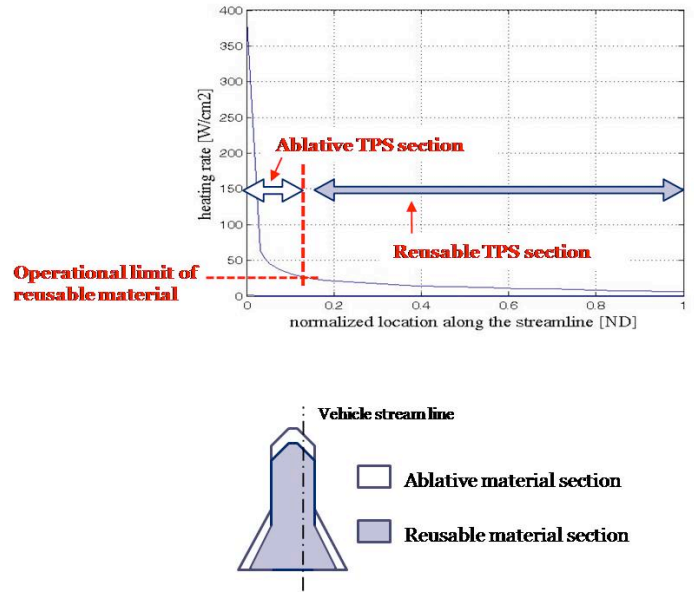

Figure 3. Schematic of TPS area definition 


\section{III.B. Required TPS Thickness Sizing}

In this section we determine the TPS thickness required for the positions of maximum heat load. For the ablative TPS section this occurs for the stagnation heating rate profile and for the reusable section it occurs for the heating rate profile peaking at the operational limit of the material, $31.9 \mathrm{~W} / \mathrm{cm}^{2}$.

To determine the maximum TPS thickness of the LI-900 section, where the heating rate is at the operational limit of LI-900, $31.9 \mathrm{~W} / \mathrm{cm}^{2}$, we utilized a transient finite element (FE) heat transfer model. The heat transfer problem is modeled as shown in Fig. 4.

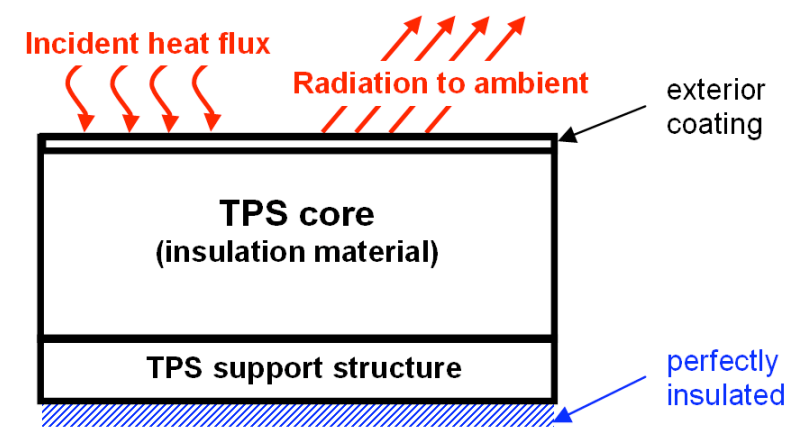

Figure 4. Schematic representation of the thermal problem

The TPS is comprised of the main core material (LI-900 in this case) which is usually covered by a thin exterior coating. This coating, reaction cured glass (RCG) in our case, acts mainly as an emissivity enhancing and oxidation protection layer. The core TPS material is also usually attached through a strain isolation pad to a TPS support structure. The TPS support structure is assumed perfectly insulated, which is a worst case assumption, since if heat could leak through the support structure the maximum temperature would decrease, becoming less critical. In our case we assume an aluminum support structure $0.254 \mathrm{~cm}$ thick typical for this type of TPS. ${ }^{24}$ Note that this represents a homogenized thickness. In reality we would have a thinner skin reinforced with stiffeners. The exterior TPS surface is subject to an incident heating rate and outgoing radiation. The incident heating rate is the stagnation point heating rate for the corresponding trajectory scaled down to $31.9 \mathrm{~W} / \mathrm{cm}^{2}$ maximum. This problem is modeled as a one-dimensional transient finite element heat transfer problem using the commercial FE software Abaqus ${ }^{\circledR}$. Fifty-four three node heat transfer link elements were used for the three sections of the TPS (exterior RCG coating, LI-900 TPS core, Aluminum support structure). Temperature dependent material properties from the TPSX material database $^{20}$ and Ref. 21 were used for all sections. After the end of the incident heating rate the simulation is continued with an additional time step where only radiation is assumed at the top surface. This is because the maximum support structure temperature peaks well after the end of the incident heating rate. Using this FE model the thickness of the LI-900 insulation required was sized. Successive analyses were carried out with variable insulation thickness until the minimum thickness was found that allowed the maximum support structure temperature to be $450 \mathrm{~K}$ or less, which is maximum operational temperature of Aluminum.

To calculate the thickness of the ablative material at the stagnation point we used a simplified analysis tool called the virtual ablation method. ${ }^{13}$ The key concept of this method is to divide a total heat load into two components as shown in Fig. 5. One is called the ablation heat load, $Q_{A}$, which makes a contribution only to the ablation process. The other is the conduction heat load, $Q_{C}$, which consists of the heat conducted into the TPS (accounting for the heat capacity of the TPS, the heat influx and the heat radiated away from the surface). Each of these heat loads determines a certain thickness, the thickness of the ablated material and the thickness of the ablative insulation respectively as shown in Fig. 5. To apply this procedure we need to determine the conduction heating rate profile, which would maintain the TPS surface at ablation temperature. To obtain the value of this heating rate, $\dot{Q}_{c}$, we carried out repeated heat-transfer analyses using a FE model similar to that described in the previous subsection. For more details on the virtual ablation method refer to Ref. 13 .

To obtain the total ablative TPS thickness we need to sum up the insulation thickness that is required to maintain the support structure below the operational temperature limit $(450 \mathrm{~K})$ under the conduction 


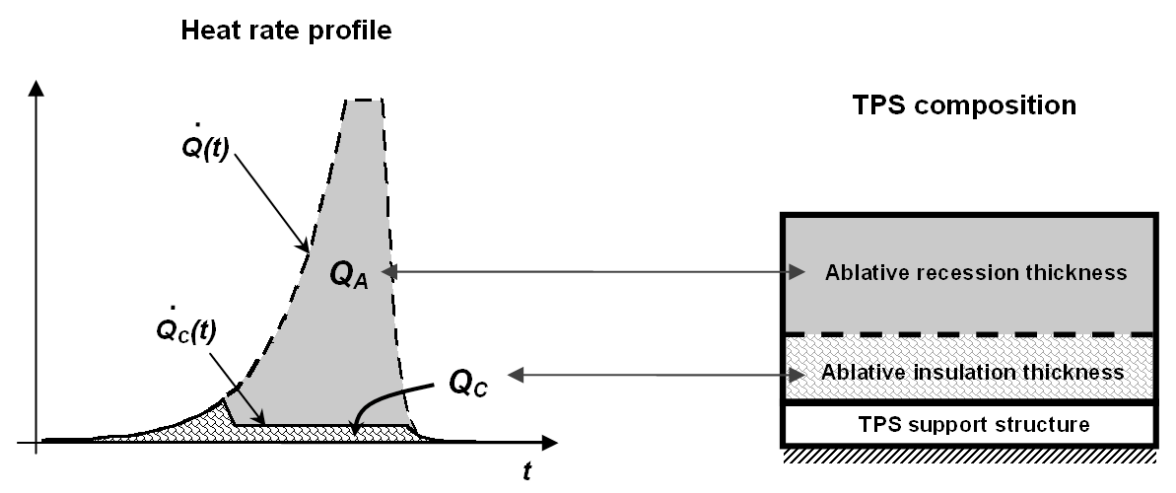

Figure 5. Virtual ablation methods

heating rate, $\dot{Q}_{c}$ alone, plus the thickness of the material that is ablated, i.e. the recession thickness, which is determined by $Q_{A}$. (cf. Fig. 5).

To determine the insulation thickness, we use the conduction heat load as input to a FE heat transfer model similar to that used for the LI-900 TPS sizing. We iteratively determined for the incident heating rate $\dot{Q}_{c}$ and the TPS thickness, which keeps the support structure of the TPS under operational temperature limit $(450 \mathrm{~K})$. Temperature dependent material properties are used such that the properties of the char layer and those of the virgin layer are combined into a single material. We finally calculate the ablative recession layer thickness, $\delta$, which can be calculated by using the heat of vaporization, $h_{v}$, and the ablation heat load, $Q_{A}$, through Eq. (25).

$$
\delta=\frac{Q_{A}}{h_{v}}
$$

$h_{v}$ in $\mathrm{J} / \mathrm{m}^{3}$ is calculated by the density of the material, $\rho_{a b l}$, and the heat of ablation in $\mathrm{J} / \mathrm{kg}, h_{a}$, as following equation and is obtained as shown in Table 4.

$$
h_{v}=\rho_{a b l} \cdot h_{a}
$$

Table 4. Heat of ablation

\begin{tabular}{ll} 
Ablative material & Heat of ablation \\
\hline SLA-561 & $1.43 \times 10^{10} \mathrm{~J} / \mathrm{m}^{3}$ \\
AVCOAT-5026-39H/CG & $1.26 \times 10^{10} \mathrm{~J} / \mathrm{m}^{3}$ \\
\hline
\end{tabular}

Note that in the procedure we use to calculate the ablative insulation thickness we assume that the recession layer does not provide insulation. This is a conservative assumption because the recession thickness for our application is high.

\section{III.C. Total TPS Mass calculation}

In the previous subsection we calculated the TPS thicknesses at the maximum heat load points for each section. To calculate the mass of the entire TPS we use the average thickness over the vehicle surface for each of the two TPS sections. This is done in the following way.

According to the method we use for the calculation of the recession thickness of the ablative TPS, the recession thickness is proportional to the heat load (See Eq. ( 25)). The thickness of the insulation part of the ablative TPS and the reusable material are calculated by one-dimensional transient finite element heat transfer model described in previous section. Fig. 6 shows a plot of the required thickness of all the materials vs. the total heat load of the AOTV mission we consider. From this plot we can see that a linear approximation would also suffice to determine the insulation thicknesses. Thus we can use an assumption that the required TPS thicknesses are linear to the total heat loads. This assumption allows us to calculate 
the average thickness over the vehicle very easily by using the average heat load over the vehicle.

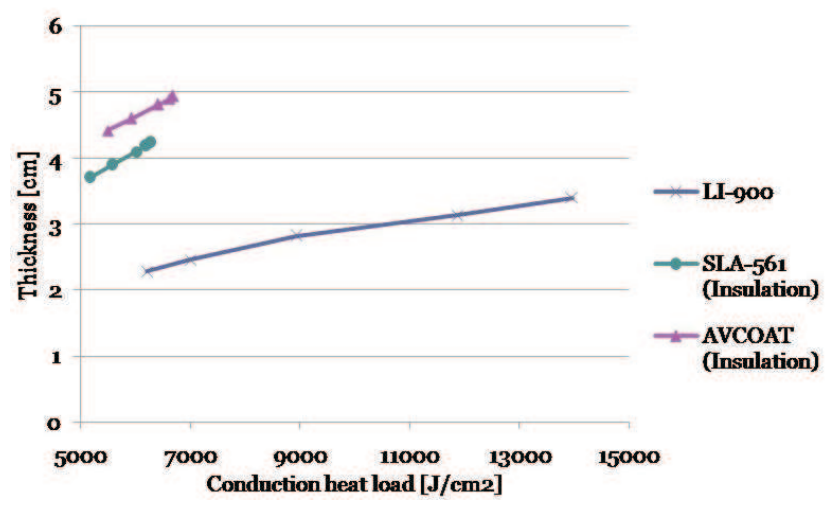

Figure 6. Required TPS thickness vs. total heat load

For the ablative TPS section, we obtain the average thickness by multiplying the maximum thickness, $T_{a b l, \max }$, by the ratio between the average heat load with respect to the streamline location of the ablative TPS section (obtained from Fig. 2), $Q_{a b l, a v e}$, and the maximum heat load (for which the maximum thickness was calculated), $Q_{a b l, m a x}$. Finally by multiplying the average thickness by the density, $\rho_{a b l}$, and the area over which the ablative material is deployed, $S_{a b l}$, we can obtain the total mass of the ablative TPS over the vehicle, $m_{a b l}$ as in Eq. (27).

$$
m_{a b l}=T_{a b l, \max } \cdot \frac{Q_{a b l, a v e}}{Q_{a b l, \max }} \cdot \rho_{a b l} \cdot S_{a b l}
$$

A similar approach is used for the reusable section, the LI-900. Using the maximum thickness of the LI-900 section, $T_{\text {reu,max }}$, which corresponds to the stagnation point heating rate scaled down to $31.9 \mathrm{~W} / \mathrm{cm}^{2}$ (the maximum heating rate for which LI-900 can still be used), we obtain the average thickness by multiplying by the ratio between the average heat load of the reusable TPS section (obtained from Fig. 2), $\rho_{\text {reu,ave }}$, and the maximum heat load (for which the maximum thickness was just calculated), $q_{\text {reu,max }}$. Multiplying by the density, $\rho_{r e u}$, and the corresponding area, $S_{r e u}$, we obtain the LI-900 TPS mass, $m_{r e u}$, as shown in Eq. $(28)$.

$$
m_{\text {reu }}=T_{\text {reu,max }} \cdot \frac{Q_{\text {reu,ave }}}{Q_{\text {reu, } \text { max }}} \cdot \rho_{\text {reu }} \cdot S_{\text {reu }}
$$

Finally by adding all the mass of the TPS, the ablative section, the reusable section and the support structure (aluminum), we can obtain the total TPS mass for the AOTV.

\section{Results and Discussion}

\section{IV.A. Trajectories of Aeroglide}

The aeroassisted orbital plane change optimal control problem described in the previous section was solved with $i_{d}=18 \mathrm{deg}$ and $\dot{Q}_{\max }=(397,454,568,681) \mathrm{W} / \mathrm{cm}^{2}\left[=(350,400,500,600) \mathrm{BTU} / \mathrm{ft}^{2} \cdot \mathrm{s}\right] \quad$ Figure 7 shows the altitude as a function of time. We see that with high heating rate constraints, the vehicle can dip deeper into the atmosphere and use the higher aerodynamic loads to stay in the atmosphere for a shorter time. This results in a smaller heat load as shown in Fig. 8 and Table 5 . Table 5 shows also the $\Delta V$ 's and the fuel consumption for each heating rate constraint case. All cases require much less fuel than the all-propulsive maneuver, where the required $\Delta V$ is $2439.2 \mathrm{~m} / \mathrm{s}$, and the corresponding fuel consumption is $2702.8 \mathrm{~kg}$. For the aerosassited maneuvers the fuel consumption increases for lower heating rate constraints. It is mainly caused by the difference in the magnitude of $\Delta V_{2}$. As seen in Eq. (1), $\Delta V_{2}$ mainly depends 
on the velocity at the exit of the atmosphere, $v_{f}$, and in the most fuel consuming case, where the heating rate constraint is $397 \mathrm{~W} / \mathrm{cm}^{2}$, it can be seen that the value of $v_{f}$ is the smallest (Fig. 9). This is because due to the low heating rate constraint the vehicle has to fly longer through the dense atmosphere since the lateral aerodynamic force for the inclination change is limited, meaning that it is exposed longer to a high drag force. This reduces most the velocity at the exit of the atmospheric flight and accordingly the fuel consumption is the highest for the lowest heating rate constraint.

An interesting feature in terms of TPS design is that when the heating rate constraint is lowered the total heat load, which is the key factor to determine the required TPS thickness, increases. This is related to the effect we mentioned earlier. When the heating rate is further constrained the lateral aerodynamic force for the inclination change is limited so that the vehicle has to fly longer at its maximum heating rate where the vehicle can generate the largest aerodynamic force (Fig. 8).

Table 5. Fuel consumption and heat load

\begin{tabular}{cccccc}
\hline $\begin{array}{c}\dot{Q}_{\max } \\
\mathrm{W} / \mathrm{cm}^{2}\end{array}$ & $\begin{array}{c}\Delta V_{1} \\
\mathrm{~m} / \mathrm{s}\end{array}$ & $\begin{array}{c}\Delta V_{2} \\
\mathrm{~m} / \mathrm{s}\end{array}$ & $\begin{array}{c}\Delta V_{3} \\
\mathrm{ft} / \mathrm{s}\end{array}$ & $\begin{array}{c}\text { Fuel consumption } \\
\mathrm{kg}\end{array}$ & $\begin{array}{c}\text { Total heat load } \\
\mathrm{J} / \mathrm{cm}^{2}\end{array}$ \\
\hline 397 & 35.8 & 1641.9 & 16.6 & 2088.9 & 174070 \\
454 & 32.9 & 1448.3 & 16.6 & 1901.8 & 168987 \\
568 & 30.3 & 1247.8 & 16.6 & 1695.3 & 159006 \\
681 & 28.9 & 1189.6 & 16.6 & 1632.1 & 149559 \\
no limit & 28.7 & 1185.5 & 16.6 & 1627.6 & 148915 \\
All-propulsive case & & 2439.2 & & 2702.8 & 0 \\
\hline
\end{tabular}

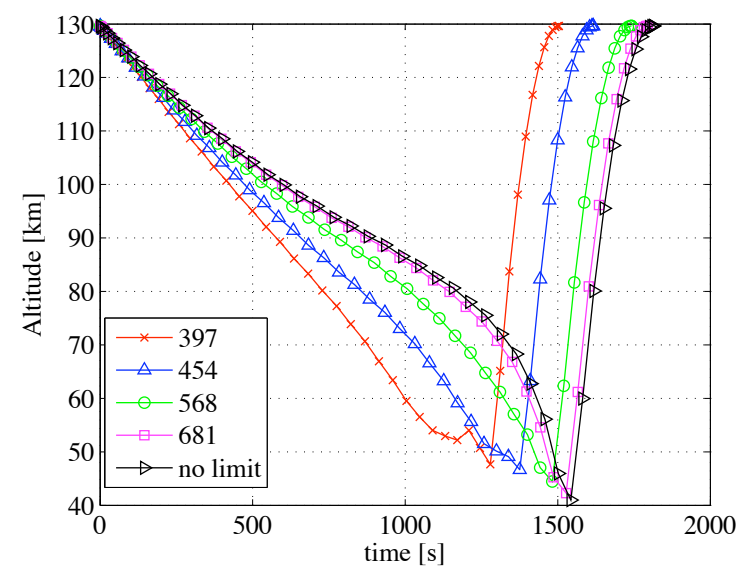

Figure 7. altitude vs time.

\section{IV.B. TPS Mass Estimation}

Table 6 shows the maximum heat load and the maximum thickness for LI-900 section. The lower the heating rate constraint the thicker the maximum thickness of the LI-900 section corresponding to the total heat load.

The heat load distribution for the ablative TPS sizing is obtained as shown in Table 7 and the required maximum insulation thickness and the recession thickness for the ablative TPS and maximum thickness for the LI-900 are given in Table 8. Similarly to the total heat load, the ablative heat load increases as the heating rate constraint decreases. This results in the maximum recession thickness of ablative TPS getting thicker as the limit on heating rate gets lower. Comparing the SLA-561 and AVCOAT-5026-39H/CG sections, the insulation thickness of AVCOAT-5026-39H/CG is slightly thicker than that of the SLA-561 due to mainly the relatively high thermal conductivity of AVCOAT-5026-39H/CG. The recession layer thicknesses of both materials are similar since their heat of ablation are close to each other (See Table 4). 


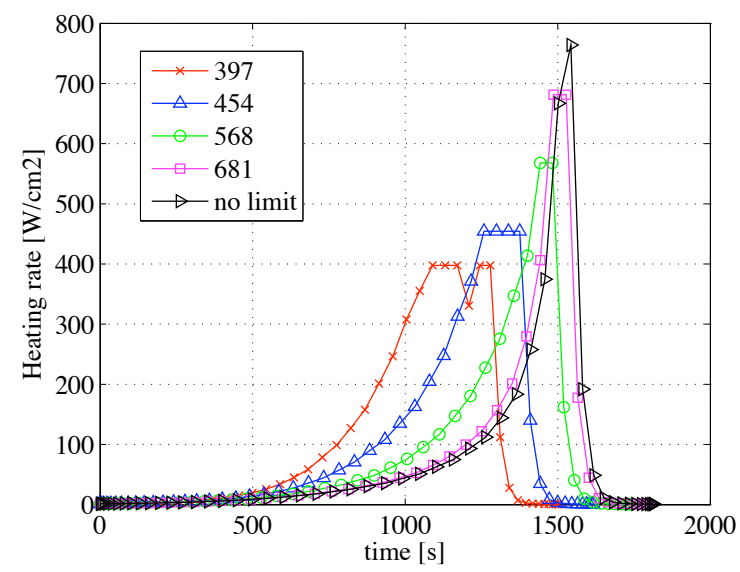

Figure 8. heating rate vs time.

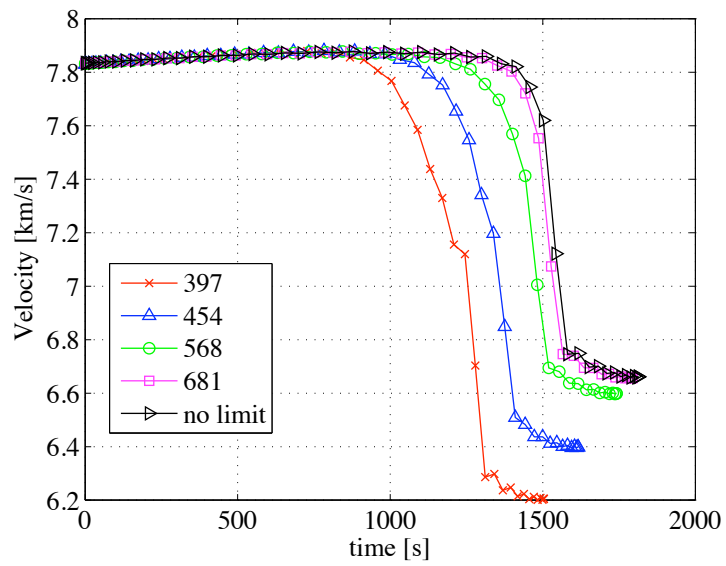

Figure 9. velocity vs time.

Table 6. Maximum heat load and thickness of LI-900

\begin{tabular}{llrrrr}
\hline Constraints $\left[\mathrm{W} / \mathrm{cm}^{2}\right]$ & no limit & 681 & 568 & 454 & 397 \\
\hline Total heat load $\left[\mathrm{J} / \mathrm{cm}^{2}\right]$ & 6219 & 7004 & 8939 & 11871 & 13975 \\
Thickness $[\mathrm{cm}]$ & 2.28 & 2.46 & 2.82 & 3.13 & 3.40 \\
\hline
\end{tabular}

Table 7. Heat load distribution for ablative TPS sizing

\begin{tabular}{llrrrrr} 
& & & & & & {$\left[\mathrm{J} / \mathrm{cm}^{2}\right]$} \\
\hline Constraints $\left[\mathrm{W} / \mathrm{cm}^{2}\right]$ & no limit & 681 & 568 & 454 & 397 \\
\hline & Total heat load & 148915 & 149559 & 159006 & 168987 & 174070 \\
\hline SLA-561 & Ablation heat load & 142639 & 143350 & 152979 & 163408 & 168896 \\
$\left(\dot{Q}_{c}=3.81 \mathrm{~W} / \mathrm{cm}^{2}\right)$ & Conduction heat load & 6576 & 6209 & 6027 & 5579 & 5174 \\
\hline AVCOAT-5026 & Ablation heat load & 142223 & 142940 & 152589 & 163054 & 168570 \\
$\left(\dot{Q}_{c}=4.02 \mathrm{~W} / \mathrm{cm}^{2}\right)$ & Conduction heat load & 6692 & 6619 & 6417 & 5933 & 5500 \\
\hline
\end{tabular}


Table 8. Maximum thickness of ablative TPS

\begin{tabular}{llrrrrr} 
& & & & & & {$[\mathrm{cm}]$} \\
\hline Constraints $\left[\mathrm{W} / \mathrm{cm}^{2}\right]$ & & no limit & 681 & 568 & 454 & 397 \\
\hline SLA-561 & Insulation thickness & 4.23 & 4.19 & 4.10 & 3.89 & 3.70 \\
& Recession thickness & 9.99 & 10.04 & 10.71 & 11.44 & 11.83 \\
& Total thickness & 14.22 & 14.23 & 14.81 & 15.33 & 15.53 \\
AVCOAT-5026 & Insulation thickness & 4.94 & 4.90 & 4.81 & 4.61 & 4.42 \\
& Recession thickness & 11.29 & 11.35 & 12.12 & 12.95 & 13.38 \\
& Total thickness & 16.23 & 16.25 & 16.93 & 17.56 & 17.80 \\
\hline
\end{tabular}

An interesting feature is that the recession thickness of the ablative section is larger than the required insulation which can keep the support structure of the TPS under the operational temperature limit . This trend is not seen in the past experiences of reentry mission, which used ablative materials for heat shielding such as the Apollo mission and the Stardust mission. In the past missions, the recession thicknesses of the materials are smaller compared to the required thickness for heat shielding, i.e., $4.8 \mathrm{~cm}$ total ablative TPS thickness with $1.2 \mathrm{~cm}$ of recession for the Stardust mission, ${ }^{25} 0.9$ in thickness with about 0.1 in of recession for the Apollo Flight AS 202. ${ }^{19,26}$ This is because the AOTV maneuver lasts much longer than typical reentry maneuver in order to achieve the required orbital plane change (i.e., about $800 \mathrm{~s}$ with $22731 \mathrm{~J} / \mathrm{cm}^{2}$ $\left(20000 \mathrm{BTU} / \mathrm{ft}^{2}\right)$ for the Apollo mission ${ }^{27}$ and about $80 \mathrm{~s}$ with $28000 \mathrm{~J} / \mathrm{cm}^{2}$ for the Stardust mission ${ }^{25}$ ), thus making the recession thickness much larger as explained below. Commonly for the reentry missions, in order to take advantage of ablative materials, with a high temperature resistance and a low thermal diffusivity which can block a sudden impact of high heating rate, its trajectory is designed to reduce the total heat load by taking the highest possible heating rate and making the mission time shorter. By reducing the total heat load it can avoid the disadvantage of its high thermal conductivity which might cause an excessive conduction heat transfer to the support structure. However, in such a severe heating environment of the aeroassisted orbital plane change, where both the total heat load and the heating rate are quite high, it can be seen that the recession layer of the ablative material has to be quite large as well.

The results for the area definition and mass estimation are given in Table 9-11 respectively. The mass of the LI-900 varies corresponding to the maximum required thickness (i.e., the thicker the maximum thickness the heavier the total LI-900 mass). For the ablative material the trend is different. This is because the area for ablative material significantly reduces as the heat limit gets lower. Consequently the total mass of the TPS, summing up masses of ablative material, LI-900 and support structure, doesn't vary much with respect to the total heat load, even though the maximum required thicknesses for each section varies significantly with the respective heat load.

Table 9. Area definition

\begin{tabular}{llrrrr} 
& & & & {$\left[\mathrm{m}^{2}\right]$} \\
\hline Constraints $\left[\mathrm{W} / \mathrm{cm}^{2}\right]$ & no limit & 681 & 568 & 454 & 397 \\
\hline Ablative material & 13.73 & 11.44 & 7.72 & 5.22 & 4.17 \\
Reusable material & 28.76 & 31.05 & 34.77 & 37.27 & 38.32 \\
\hline
\end{tabular}

Table 10. TPS Mass for SLA-561

\begin{tabular}{|c|c|c|c|c|c|}
\hline & & & & & {$[\mathrm{kg}]$} \\
\hline Constraints $\left[\mathrm{W} / \mathrm{cm}^{2}\right]$ & no limit & 681 & 568 & 454 & 397 \\
\hline SLA-561 & 52.8 & 49.1 & 43.8 & 39.5 & 37.2 \\
\hline LI-900 & 59.4 & 64.6 & 75.7 & 77.3 & 78.1 \\
\hline Total $\operatorname{TPS}^{(*)}$ & 410.8 & 412.4 & 418.3 & 415.5 & 413.9 \\
\hline
\end{tabular}

(*)including support structure 
Table 11. TPS mass for AVCOAT-5026

\begin{tabular}{|c|c|c|c|c|c|}
\hline & & & & & {$[\mathrm{kg}]$} \\
\hline Constraints $\left[\mathrm{W} / \mathrm{cm}^{2}\right]$ & no limit & 681 & 568 & 454 & 397 \\
\hline AVCOAT-5026 & 120.6 & 112.0 & 99.4 & 88.6 & 82.5 \\
\hline LI-900 & 59.4 & 64.6 & 75.7 & 77.3 & 78.1 \\
\hline Total $\operatorname{TPS}^{(*)}$ & 478.7 & 475.3 & 473.9 & 464.6 & 459.3 \\
\hline
\end{tabular}

$(*)$ including support structure

Table 12-13 shows a mass gain for each case. The amount of fuel saving is obtained by comparing the original fuel carrying capacity, $2614.8 \mathrm{~kg}$ (Table 1), to the required fuel consumption for the inclination change and the total mass gain is calculated by subtracting the TPS mass from the fuel saving. This mass gain is equivalent to an additional available payload mass since we have made the assumption that the total weight and the exterior shape of the vehicle are fixed. The total mass gain for the best case (no limit case) is about $600 \mathrm{~kg}$ compared to that of the all-propulsive maneuver. This means that even when considering the mass penalty induced by the TPS requirement the aeroassisted maneuver still presents a substantial mass advantage over an all-propulsive maneuver. In addition, for designing the lightest vehicle with a fixed payload mass, the mass gain we obtain will be amplified. The mass saving would bring a further mass reduction since it would allow for a smaller and lighter vehicle that requires. We also used a relatively simple TPS mass estimation model which we tried to make conservative. The main conservative assumptions are: neglecting the insulation role of the recession thickness in the the virtual ablation method; assuming that the heating rate distribution over the vehicle length does not vary along the vehicle width (in reality the heating rate decreases towards the tip of the wings); assuming the heating rate distribution is independent of the angle of attack (the distribution used is for high angle of attack involving high heating). The main remaining unconservative assumptions are: neglecting the radiative heat flux contribution; neglecting the leeward side TPS. Accordingly the exact TPS mass and so the corresponding mass savings might change if a detailed TPS sizing analysis was carried out.

Table 12. Mass gain for SLA-561

\begin{tabular}{lrrrrrr} 
& & & & & & {$[\mathrm{kg}]$} \\
\hline Constraints $\left[\mathrm{W} / \mathrm{cm}^{2}\right]$ & no limit & 681 & 568 & 454 & 397 & all-propulsive \\
\hline Fuel saving & 987.4 & 982.8 & 919.6 & 713.2 & 526.0 & -87.9 \\
Total TPS mass & 410.8 & 412.4 & 417.8 & 414.6 & 412.7 & 0 \\
\hline Total mass gain & 576.6 & 570.7 & 501.8 & 298.6 & 113.3 & -87.9 \\
\hline
\end{tabular}

Table 13. Mass gain for AVCOAT-5026

\begin{tabular}{lrrrrrr} 
& & & & & & {$[\mathrm{kg}]$} \\
\hline Constraints $\left[\mathrm{W} / \mathrm{cm}^{2}\right]$ & no limit & 681 & 568 & 454 & 397 & all-propulsive \\
\hline Fuel saving & 987.4 & 982.8 & 919.6 & 713.2 & 526.0 & -87.9 \\
Total TPS mass & 478.7 & 475.3 & 473.9 & 464.6 & 459.3 & 0 \\
\hline Total mass gain & 508.7 & 507.5 & 445.7 & 248.6 & 66.7 & -87.9 \\
\hline
\end{tabular}

We now examine the impact of the TPS mass on the overall lightest vehicle (i.e., total mass gain). Intuitively one expects that the higher the heating rate constraint the severer the thermal reentry environment so the more TPS is required. In this case fuel consumption and TPS mass have opposite effects on the total mass of the vehicle relative to the heating rate constraint: from a fuel consumption point of view alone the minimum mass would be achieved with no heating rate constraint. From a TPS point of view alone the minimum mass would be achieved aeroassist with the lowest possible heating rate constraint allowing the required inclination change. We would then expect that the minimum overall mass would be achieved somewhere between the aeroassisted maneuver with no heating rate constraint and the one with the lowest 
possible heating rate constraint.

However the results show that this is not the case, the minimum mass vehicle (the maximum mass gain) being still obtained for the no heating rate constraint case, i.e., the optimal case when doing a trajectory optimization alone without taking the TPS size into account. Indeed the variations of the TPS mass from one heating rate constraint case to the other are too small compared to the fuel saving variations to change the trend of the total mass gain. We explained earlier why the TPS mass doesn't necessarily increase with the heating rate constraint leading to small TPS mass variations.

Note however that the difference of the total mass gain between the $681 \mathrm{~W} / \mathrm{cm}^{2}$ and the no limit heating rate constraint cases is relatively small. Considering the simplified TPS mass estimation model used, this difference is close to the error bounds of our model. If a detailed TPS sizing was carried out one or the other heating constraint case could be shown to be the optimum. These two heating rate constraint cases are however very close to each other from a trajectory optimization point of view (see Fig. 7-9). From a mass point of view as well, using one or the other case leads to very similar results, so we chose to consider the no limit case as the optimum in all the cases since the unconstrained approach is the easiest to implement.

Two assumptions may have limited the fuel savings. First, the inclination change was done entirely via the aeroassist, without any propulsive inclination change. For low heat rate constraints it is probably more efficient to have some propulsive inclination change. Second, the coupling between the trajectory optimization and the TPS design was limited to a single parameter, the heat rate constraint. Since the mass of the TPS depends also on the total heat load, further savings may be realized by using a two parameter coupling with both heat rate and total heat load constraints. For the present example, we do not expect that this second parameter will contribute much to the mass savings, but for other vehicles, a two-parameter coupling may.

\section{Conclusion}

This article presented a mass trade-off for an aeroassisted orbital transfer vehicle (AOTV) including both fuel mass and thermal protection system (TPS) mass. We analyzed for an aeroglide maneuver the effect of the coupling existing between the trajectory and the thermal protection system size through a single coupling parameter, the maximum heating rate constraint. We considered a one way coupling, assuming that the total weight and the exterior shape of the vehicle are fixed, which allowed us to construct a simple sequential MDO framework. We first carried out a trajectory optimization which optimized all guidancerelated parameters along the trajectory, including de-orbit, boost and recircularization impulses to determine the heating rate profiles for the different maximum heating rate constraints. Then in a second step a simplified mass estimation model was used to size the TPS for each heating rate profile. This model mapped the vehicle surface with and ablative or reusable materials according to the heating rate at the corresponding vehicle location. The reusable TPS section was then sized using a transient heat transfer finite element model. The ablative TPS section was sized using the virtual ablation method.

It was found that even when considering the TPS mass an aeroassisted maneuver maintains a mass advantage over an all-propulsive maneuver. The total TPS mass was however found to vary relatively little relative to the heating rate constraint. This resulted in the TPS mass variations being small compared to the fuel saving variations. Accordingly the minimum mass AOTV (including both fuel and TPS masses) was found to be achieved if no heating rate constraint was imposed, which is also the scenario which minimizes the fuel consumption alone.

The presented MDO framework is general and can be potentially applied to different vehicles, aeroassisted orbital transfer scenarios or TPS mass estimation models as long as the assumption is made that the achieved mass savings are transformed into additional payload capacity so that the total mass and shape of the vehicle remain constant.

\section{Appendix - Optimal Control of Trajectory Optimization -}

Examining the detail of the trajectories, Table 14 shows the numerical results of these trajectories such as the initial velocity, $v_{i}$, initial flight path angle, $\gamma_{i}$, final velocity, $v_{f}$, final flight path angle, $\gamma_{f}$, and maximum velocity during the descent phase, $v_{\max }$. Fig. 10-12 show the profile of the control variables, angle of attack, $\alpha$, and bank angle, $\sigma$. The slightly steeper initial flight path angle is applied to the most constrained case, 397 $\mathrm{W} / \mathrm{cm}^{2}$ case. A steeper flight can take an advantage of using the gravity efficiently to increase the velocity 
during the descent phase, even though making the initial flight path angle steeper has a disadvantage on the fuel consumption such as the larger deorbit impulse and the smaller initial entry velocity according to Kepler's laws of planetary motion. Consequently the steeper flight can restore the velocity (See $v_{\max }$ ) in descent phase.

In all cases the atmospheric flight starts with the angle of attack of about $35 \mathrm{deg}$ and the bank angle of about $-180 \mathrm{deg}$. At the beginning of the descent phase where the air density is quite small, a high angle of attack and a bank angle of about $-180 \mathrm{deg}$ are needed to generate a downward force in order to decrease the altitude. During the descent phase, it can be seen that the angle of attack varies actively. Generally, in terms of maintaining the velocity, making the angle of attack smaller corresponding to the altitude decrease is preferable since a high angle of attack also leads to a high drag force. On the other hand, generating as a large lateral force as possible by taking a high angle of attack with a certain bank angle is needed to achieve the desired inclination change quickly. The jaggedness of the angle of attack in the numerical solution is most likely due to this trade-off. This behavior is numerical noise since the air density in the descent phase is quite small to generate a meaningful aerodynamic force. (In other words, the behavior of the angle of attack has to be big to generate a certain aerodynamic force in such a quite thin atmosphere.)

Table 14. Numerical results for aeroglide trajectories

\begin{tabular}{cccccc}
\hline$\dot{Q}_{\max }$ & $\gamma_{i}$ & $v_{i}$ & $\gamma_{f}$ & $v_{f}$ & $v_{\max }$ \\
$\mathrm{W} / \mathrm{cm}^{2}$ & $\mathrm{deg}$ & $\mathrm{m} / \mathrm{s}$ & $\mathrm{deg}$ & $\mathrm{m} / \mathrm{s}$ & $\mathrm{m} / \mathrm{s}$ \\
\hline 397 & -0.523 & 7827.3 & 0 & 6204.4 & 7879.8 \\
454 & -0.482 & 7830.2 & 0 & 6398.1 & 7880.8 \\
568 & -0.442 & 7832.7 & 0 & 6598.6 & 7878.1 \\
681 & -0.419 & 7834.1 & 0 & 6656.7 & 7876.9 \\
no limit & -0.416 & 7834.3 & $1.6 \times 10^{-3}$ & 6660.8 & 7877.6 \\
\hline
\end{tabular}

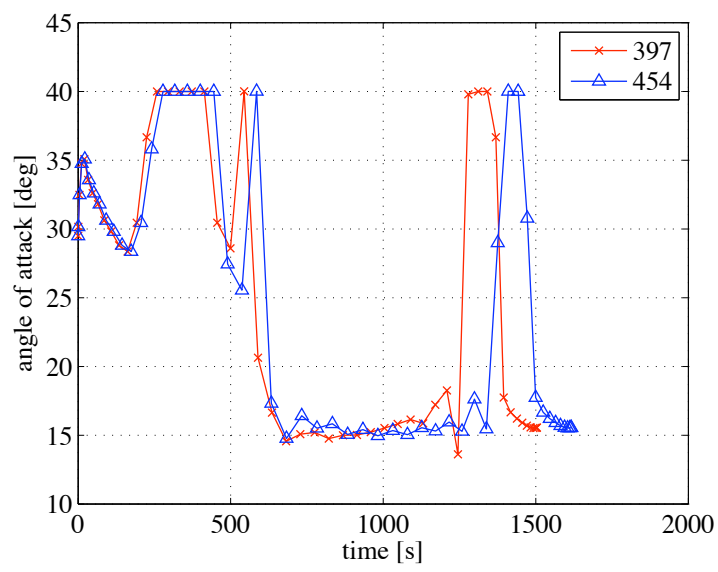

Figure 10. Angle of attack vs time (1/2).

Once the altitude decreases enough to get a sufficient aerodynamic force to change the inclination (at an altitude of around $70 \mathrm{~km}$ ), the angle of attack remains at around $16 \mathrm{deg}$, which corresponds to the maximum $L / D$. It allows the vehicle to fly as efficiently as possible without losing its velocity excessively since in this altitude region the drag force is not negligible. As the altitude approaches to its minimum, where the vehicle meets maximum aerodynamic forces and correspondingly maximum heating rates, the bank angle gets close to $-90 \mathrm{deg}$ to generate the maximum lateral force to make the inclination change. After the desired inclination is achieved, in order to go back to the initial orbit altitude the upward force is created by taking the highest angle of attack and the bank angle of close to 0 deg. 


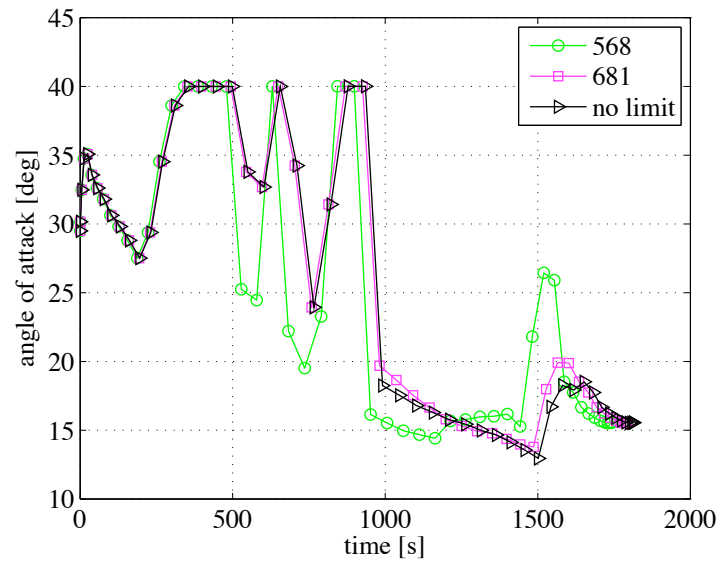

Figure 11. Angle of attack vs time (2/2).

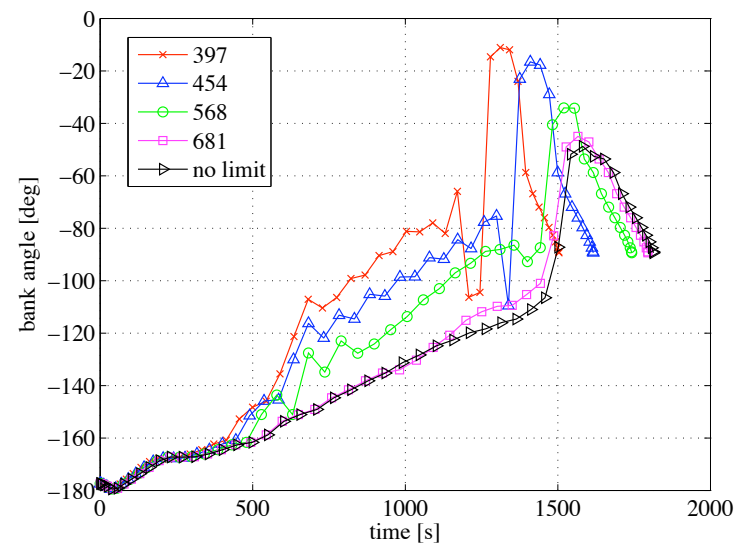

Figure 12. Bank angle vs time.

18 of 19 


\section{References}

${ }^{1}$ Stephen, A. W., Bonnie, J. D., "Orbital Space Plane, Past, Present, and Future," AIAA International Air and Space Symposium and Exposition: The Next 100 Years, AIAA Paper 2003-2718, Dayton, Ohio, July 14-17, 2003.

${ }^{2}$ Walberg, G. D., "A Survey of Aeroassisted Orbit Transfer," Journal of Spacecraft and Rockets, Vol. 22, No. 1, 1985, pp.3-18.

${ }^{3}$ Mease, K. D., "Optimization of Aeroassisted Orbital Transfer: Current Status," The Journal of Astronautical Sciences, Vol. 36, No. 1/2, 1988, pp.7-33.

${ }^{4}$ Lee, J. Y., Hull, D. G., "Maximum Orbit Plane Change with Heat-Transfer-Rate Considerations," Journal of Guidance, Control, and Dynamics, Vol. 13, No. 3, 1990, pp.492-497.

${ }^{5}$ Seywald, H, "Optimal Control Solutions for an Aeroassisted Orbital Transfer Problem with a Heating Rate Limit," AIAA Guidance, Navigation and Control Conference, AIAA Paper 1994-3647, Scottsdale, AZ, Aug 1-3, 1994.

${ }^{6}$ Nicholson, J. C., and Ross, I. M., "Performance of Optimal Synergetic Maneuvers," Advances in the Astronautical Sciences, AAS Paper 95-120, Vol. 89, Pt. 2, Univelt, San Diego, CA, 1995, pp. 923-935.

7Zimmermann, F., Calise, A. J., "Numerical Optimization Study of Aeroassisted Orbital Transfer," Journal of Guidance, Control and Dynamics, Vol. 21, No. 1, 1998, pp. 127-133.

${ }^{8}$ Rao, A. V., Tang S., and Hallman, W. P. "Numerical Optimization Study of Multiple-Pass Aeroassisted Orbital Transfer," Optimal Control Applications and Methods Vol. 23, No. 4, July-August 2002, pp. 215-238.

${ }^{9}$ Rehder, J. J., "Multiple Pass Trajectories for an Aeroassisted Orbital Transfer Vehicle," AIAA Aerospace Sciences Meeting, AIAA Paper 84-0407, Reno, NV, Jan 9-12, 1984.

${ }^{10}$ Pienkowski, J., Whitmore, S., Spencer, M., "Analysis of the Aerodynamic Orbital Transfer Capabilities of the X-37 Space Maneuvering Vehicle (SMV)," Aerospace Sciences Meeting and Exhibit, AIAA Paper 2003-908, Reno, Nevada, Jan. 6-9, 2003.

${ }^{11}$ Rao, A. V., User's Manual for GPOCS Version 1.1: A MATLAB Implementation of the Gauss Pseudospectral Method, TOMLAB Optimization, Vasteras, Sweden, September 2007.

${ }^{12}$ Benson, D. A., Huntington, G. T., Thorvaldsen, T. P., and Rao, A. V., "Direct Trajectory Optimization and Costate Estimation via an Orthogonal Collocation Method," Journal of Guidance, Control, and Dynamics, Vol. 29, No. 6, NovemberDecember, 2006, pp. 1435-1440.

${ }^{13}$ Ko, W. L., Gong, L., Quinn, R. D., "Reentry Thermal Analysis of a Generic Crew Exploration Vehicle Structure," NASA TM-214607, 2007.

${ }^{14}$ Vinh, N-X, Busemann, A., and Culp, R. D., Hypersonic and Planetary Entry Flight Mechanics, University of Michigan Press, Ann Arbor, 1980.

${ }^{15}$ U.S. Standard Atmosphere, 1962, U.S. Government Printing Office, Washington, D.C., 1962.

${ }^{16}$ Detra, R. W., Kemp, N. N., and Riddell, F. R., "Addendum to "Heat Transfer to Satellite Vehicles Reentering the Atmosphere," Jet Propulsion, Vol. 27, No. 12, 1957, pp. 1256-1257.

${ }^{17}$ Holmstrom, K., Goran, A. O., and Edvall, M. M., "User's Guide for TOMLAB/SNOPT," TOMLAB Optimization, 5 July 2005.

${ }^{18}$ Gill, P. E., Murray, W., and Saunders, M. A., "SNOPT: An SQP Algorithm for Large Scale Constrained Optimization," SIAM Journal on Optimization, Vol. 12, No. 4, 2002.

${ }^{19}$ Erb, R. B., Greenshields, D. H., Chauvin, L. T., Pavlosky, J. E., "Apollo Thermal-Protection System Development," Journal of Spacecraft and Rockets, Vol. 7 No. 6, 1970, pp. 727-734

${ }^{20}$ NASA Ames Thermal Protective Materials and System Branch, TPSX Database [online database], http://tpsx.arc.nasa.gov/index.html, 26 September.

${ }^{21}$ Williams, S. D., Curry, D. M., "Thermal Protection Materials Thermophysical Property Data," NASA-RP-1289, 1992.

${ }^{22}$ Menees, G. P., Brown, K. G., Wilson, J. F., Davies, C. B.,"Aerothermodynamic Heating and Performance Analysis of a High-Lift Aeromaneuvering AOTV Concept," Journal of Spacecraft and Rockets, Vol. 24, No. 3, 1987, pp. 198-204.

${ }^{23}$ NASA Marshall Space Flight Center, "NASA Facts," May 2003, FS-2003-05-65-MSFC.

${ }^{24}$ Myers, D., Martin, C., Blosser, M. L., "Parametric Weight Comparison of Advanced Metallic, Ceramic Tile, and Ceramic Blanket Thermal Protection Systems (TPS)," NASA TM-210289, 2000.

${ }^{25}$ Olynick, D., Chen, Y. K., Tauber, M. E., "Forebody TPS sizing with radiation and ablation for the Stardust Sample Return Capsule," AIAA Thermophysics Conference, AIAA-1997-2474, Atlanta, GA, June 23-25, 1997.

${ }^{26}$ Bartlett, E. P., Abbett, M. J., Nicolet, W. E., Moyer, C. B., "Improved heat-shield design procedures for manned entry systems, part II, Application to Apollo," NASA-CR-108689, 1970.

${ }^{27}$ NASA Manned Spacecraft Center, Houston, Texas, "Postlaunch Report for Mission AS-202 (Apollo Spacecraft 011)", Report MSC-A-R-66-5, October 1966. 